\title{
Heerfordt-Syndrom
}

Eine 32-jährige Frau litt seit etwa sechs Wochen unter einer beidseitigen Schwellung im Bereich der Präaurikularregion sowie Augen- und Mundtrockenheit. Die Mimik der rechten Gesichtshälfte war beeinträchtigt, und sie spürte ein Kribbeln der rechten Zungenhälfte.

Die Untersuchung ergab eine beidseits stark vergrößerte und druckschmerzhafte Parotis. Auch die Gll. submandibulares und die Tränendrüsen waren angeschwollen (Abb. A). Die Körpertemperatur war normal. Es lag keine Uveitis vor. Der Lidschluss rechts und das Spitzen der Lippen waren nur eingeschränkt möglich, die sensiblen Facialisanteile aber beidseits intakt. Serologische Untersuchungen auf Borreliose und HIV verliefen negativ.

Eine Röntgenaufnahme des Thorax ergab beidseits vergrößerte Hiluslymphknoten. Im CT des Schädels erkannte man beidseits eine gleichmäßig kontrastmittelaufnehmende Parotis (Abb. B). In
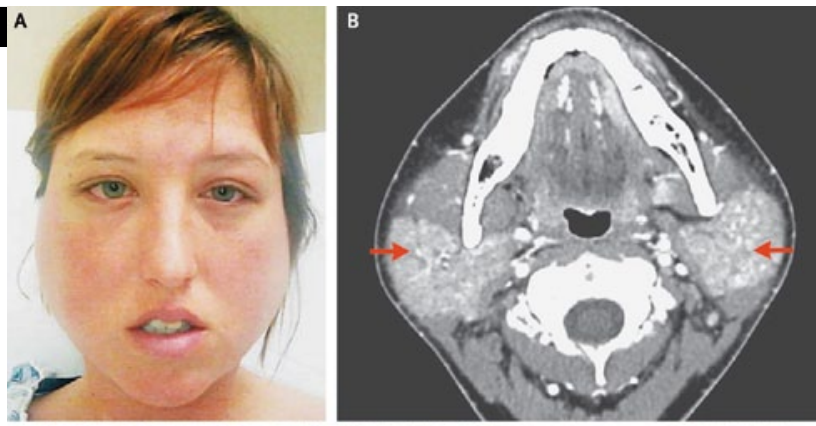
einer Biopsie aus der rechten Parotis fand man verstreut liegende Granulome mit zentralen Nekrosen. Spezialfärbungen auf säurefeste Stäbchen und Pilze waren negativ (Abb. C, HE-Färbung). Unter der Diagnose eines Heerfordt-Syndroms, einer seltenen Form der Sarkoidose mit Kompression des Facialis, wurde die Patienten mit täglich 60 mg Prednison behandelt. Bei einer Verlaufsuntersuchung 19 Tage später waren die Parotisschwellung und die Facialisparese verschwunden (Abb. D)

H. S. FüeßI =

- A.Dua und A. Manadan

Heerfordt's Syndrome

or uveoparotid fever. New Engl. J Med 2013; 269: 458

\section{Laborscreening bei kleinwüchsigen Kindern}

Asymptomatische und klinisch gesunde Kinder mit geringer Körpergröße rechtfertigen kein ungezieltes umfassendes Screening nach einer zugrunde liegenden Erkrankung.

— Nach den derzeit gültigen Leitlinien ist bei Kindern mit einer Körperlänge unterhalb der dritten Perzentile, bei denen Anamnese und körperliche Untersuchung keinen Hinweis auf eine Grunderkrankung ergeben, ein umfassendes Laborscreening auf endokrine, gastrointestinale oder chromosomale Störungen durchzuführen.

Die Krankenakten von 1373 zwischen 2008 und 2011 in einer pädiatrisch-endokrinologischen Spezialklinik vorgestellten Kinder wurden retrospektiv ausgewertet. Darunter befanden sich 235 Kinder mit einer Körperlänge unterhalb der dritten Perzentile, negativer Anamnese und normalem körperlichen Untersuchungsbefund. Nach Anwen- dung der gesamten empfohlenen Testbatterie wurde bei fast $99 \%$ dieser Kinder eine Variation des normalen Längenwachstums festgestellt.

Die Inzidenz von relevanten neu entdeckten Pathologien lag bei 1,3\%. Dabei wurden in dieser Klinik im Durchschnitt nur $64,3 \%$ der empfohlenen Untersuchungen durchgeführt. Nur 2,1\% der Patienten erhielten tatsächlich das volle Programm. Die Aufdeckung von drei neuen Erkrankungen, die möglicherweise im Zusammenhang mit dem Minderwuchs standen, wurde zudem noch erkauft mit falsch positiven Laborbefunden bei zehn Patienten. Unter den mit Deutschland kaum vergleichbaren Kostenstrukturen beliefen sich die Kosten für die gesamte Diagnostik auf über 315000 \$. Pro neue Diagnose mussten über 105000 \$ veranschlagt werden.

\footnotetext{
- S. Sisley et al.

Low incidence of pathology detection and high cost of screening in the evaluation of asymptomatic short children. J Pediatrics 2013; doi: 10.1016/j. jpeds.2013.04.002. [Epub ahead of print]
}

\section{Kommentar}

Die Untersuchung liefert ein weiteres Beispiel dafür, dass ungezieltes Laborscreening nach Erkrankungen in der Regel mehr Probleme schafft als löst. Im speziellen Fall fragt sich der unbedarfte Beobachter, warum derart anspruchsvolle Leitlinien, die selbst eine modellhafte Einrichtung nur zu zwei Drittel befolgt, überhaupt von Experten verabschiedet wurden. Man wird den Verdacht nicht los, als spielten bei der Publikation von Leitlinien nicht zuletzt ökonomische Interessen eine Rolle. Vielleicht hat sich hier einfach die "Labor-Lobby" gegen die Kliniker durchgesetzt. Das hochsensible Thema des Kleinwuchses von Kindern, bei dem besorgte Eltern die Zukunftschancen ihrer Kinder schwinden sehen, eignet sich ausgezeichnet für die Mobilisierung von Geldströmen.

H. S. FüeßI = 